\title{
Isolated subtle asymmetric slowing on EEG leading to a diagnosis of autoimmune encephalitis
}

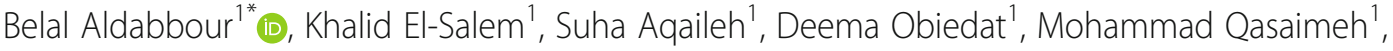 \\ Majdi Al Qawasmeh", Abdel-Hameed Al-Mistarehi ${ }^{2}$ and Ahmed Yassin ${ }^{1}$
}

\begin{abstract}
Background: Autoimmune encephalitis presents with subacute neurological, psychiatric, and behavioral symptoms. In many cases, MRI and CSF studies return normal. Laboratory testing for pathogenic neuronal antibodies is limited by accessibility and sensitivity issues. Finally, the different diagnostic approaches lack consensus and have until recently been largely retrospective. Collectively, these factors predispose for delays in diagnosis and treatment in many cases.

Case presentation: We describe the case of a 42-year-old woman who did not initially meet the newly proposed criteria for probable autoimmune encephalitis, but in whom the detection on electroencephalogram (EEG) of subtle asymmetric slowing of the left hemisphere ultimately led to the diagnosis.

Discussion: EEG is highly sensitive and rarely normal in encephalitis. The recently introduced diagnostic criteria for autoimmune encephalitis included new-onset seizures in the earliest steps in the algorithm but did not yet incorporate EEG findings. We encourage practicing physicians to use EEG as an early, sensitive diagnostic tool for cases suspected to have autoimmune encephalitis.
\end{abstract}

Keywords: Electroencephalography, Encephalitis, Paraneoplastic syndromes

\section{Background}

Encephalitis is a serious condition with the potential to cause severe and permanent disability or even death. Etiologies are diverse, and the differential diagnosis of encephalitis is often complex [1]. Generally, it may be due to infection (such as herpes simplex virus "HSV") or autoimmunity (paraneoplastic or non-paraneoplastic). Paraneoplastic encephalitis is usually caused by antibodies against intracellular neuronal proteins, while autoimmune non-paraneoplastic encephalitis in most cases is secondary to antibodies directed against neuronal cell surface or synaptic proteins $[1,2]$. The clinical

\footnotetext{
* Correspondence: belal90md@gmail.com

'Department of Neuroscience, King Abdullah University Hospital (KAUH) of Jordan University of Science and Technology (JUST), P.O. Box 630001, Irbid 22110, Jordan

Full list of author information is available at the end of the article
}

presentation is non-specific as patients with encephalitis due to infectious or autoimmune etiologies may present with variable acute or subacute neurologic and/or psychiatric manifestations, with or without fever and cerebrospinal fluid (CSF) pleocytosis [2]. Diagnosing encephalitis depends on clinical and radiological evaluation and the findings of CSF analysis, but pinpointing a definite etiology often proves to be more daunting [3]. Electroencephalography (EEG) is highly sensitive in encephalitis, especially in chronic cases, but it is rarely specific $[4,5]$. On the other hand, a normal EEG in some cases may indicate an inorganic disorder [6, 7].

\section{Case presentation}

A 42-year-old woman who is an active member of society, and who has no previous medical or psychiatric history of note, was referred to the psychiatry department

\section{Springer Open}

(๑) The Author(s). 2020 Open Access This article is licensed under a Creative Commons Attribution 4.0 International License, which permits use, sharing, adaptation, distribution and reproduction in any medium or format, as long as you give appropriate credit to the original author(s) and the source, provide a link to the Creative Commons licence, and indicate if changes were made. The images or other third party material in this article are included in the article's Creative Commons licence, unless indicated otherwise in a credit line to the material. If material is not included in the article's Creative Commons licence and your intended use is not permitted by statutory regulation or exceeds the permitted use, you will need to obtain permission directly from the copyright holder. To view a copy of this licence, visit http://creativecommons.org/licenses/by/4.0/. 
at our tertiary hospital from a peripheral center following a one-week history of an episode marked by progressively disorganized and decreased speech, social withdrawal, labile mood, persecutory delusions, irritability, agitation, decreased sleep, and fluctuating disorientation to place, person, and situation. Her best friend had recently been diagnosed with terminal lung cancer, and few days before the onset of her symptoms she suffered a severely stressful social encounter. Also, a few weeks prior to her symptoms, she suffered from a diarrheal illness that lasted nearly 10 days. Brain computed tomography $(\mathrm{CT})$ and basic laboratory investigations prior to her referral were reported as free, including a normal thyroid function, normal ESR, and lack of leukocytosis on complete blood count (CBC).

Upon admission, the patient was provisionally diagnosed as a case of acute psychosis and treated accordingly with olanzapine, alprazolam, and thiamine. However, the psychiatry team sensed that the mode and age of presentation were atypical and a warranted exclusion of organic causes. Upon evaluation by neurology, surrogate history was unrevealing of any bulbar symptoms, focal limb weakness, decreased level or loss of consciousness, convulsions, fever, or chills. A review of the medical chart revealed a mostly afebrile patient with two readings of low-grade fever (T. max 37.9 Celsius). Neurological examination revealed a disoriented and irritable patient with poverty of speech and impaired comprehension, but no focality was discerned throughout the remainder of her limited examination. A lumbar puncture was unrevealing. There was no CSF pleocytosis (white blood cell "WBC" count was zero), glucose was normal $(3.3 \mathrm{mmol} / \mathrm{l}$, serum glucose $5.3 \mathrm{mmol} / \mathrm{l})$ as well as protein $(35 \mathrm{gm} / \mathrm{dl})$. CSF gram stain, culture, cytology, and viral PCRs for HSV I and II, Mycobacterium tuberculosis (M. tb), Epstein-Barr virus (EBV), Cytomegalovirus (CMV), and enterovirus were all negative. Blood cultures were negative as well. An initial brain magnetic resonance image "MRI" used Netherlands-manufactured 3-T Philips MR Ingenia Elition (2015) and was unremarkable. Tumor markers were negative. CT of the neck, chest abdomen, and pelvis (CT-NCAP) using a 128-slice USA-made Philips ingenuity (2016) was unrevealing of hidden malignancies.

Seven days into her admission, the patient was showing no signs of improvement on antipsychotics and was increasingly agitated. A 30-min routine EEG was done using the 2011 Japan-made Nihon Kohden NeuroWorkbench. It showed left temporal polymorphic delta slowing. Also, subtle asymmetry was noted between the right and left hemispheres, with lower voltage and lack of faster frequencies over the left hemisphere (Fig. 1). Posterior dominant rhythm (PDR) was less well developed over the left side as well.

These findings prompted a repetition of brain MRI with administration of intravenous contrast, which showed evidence of left-sided enhancing leptomeninges, with enhancement of the cerebral sulci and gyri but no signs of cerebral edema (Fig. 2). The enhancement pattern correlated with the location of asymmetric slowing on EEG, further raising suspicion of a hidden organic etiology.

Repeat CSF workup was negative. Still, the patient was started empirically on ceftriaxone, vancomycin, and acyclovir. Follow up EEG 3 days later revealed left-sided $2.5 \mathrm{~Hz}$ lateralized rhythmic delta activity (LRDA), which

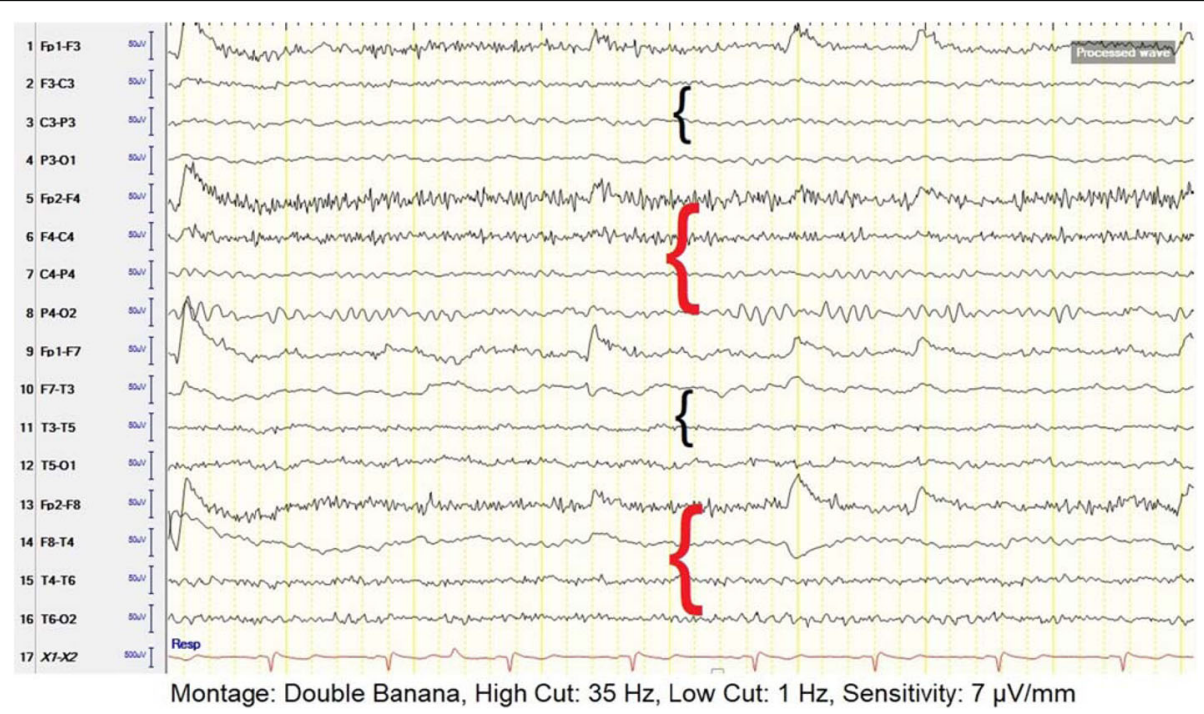

Fig. 1 EEG on presentation. EEG demonstrates a subtle asymmetry between the left (black brackets) and right (red brackets) hemispheres, with lower voltage and lack of faster frequencies over the left hemisphere 


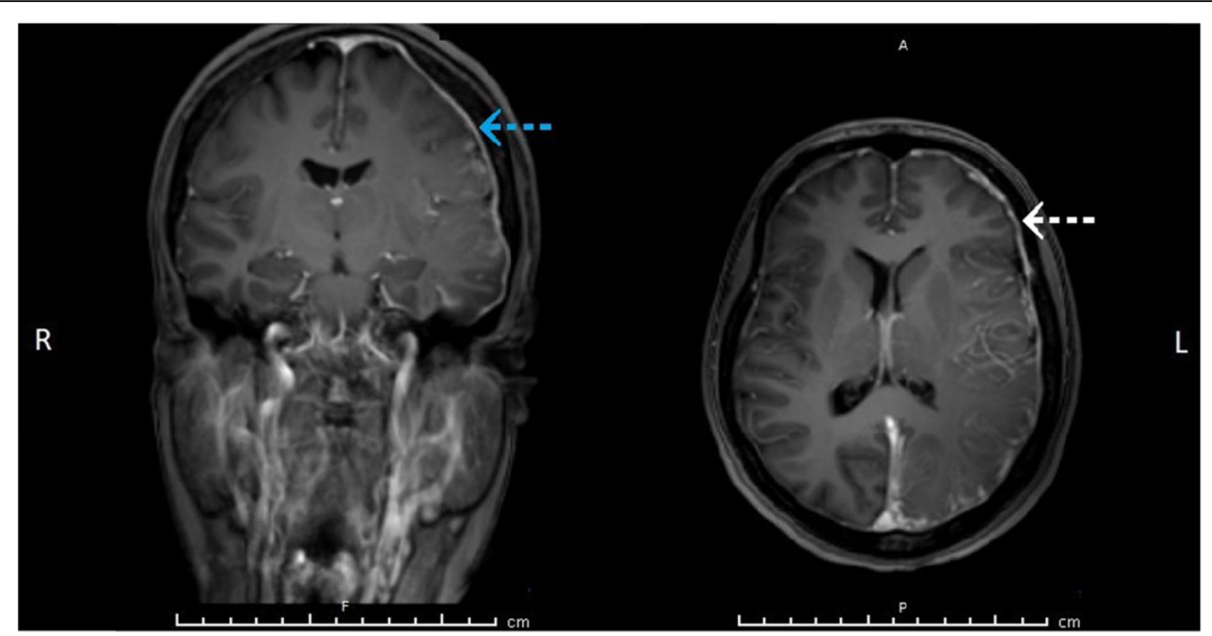

Fig. 2 Brain MRI with contrast. Coronal (left) and axial (right) T1W MRI with contrast demonstrating left-sided leptomeningeal and sulcal enhancement (arrows)

at times were preceded by a small spike, and for which levetiracetam was commenced (Fig. 3). Meanwhile, a comprehensive autoimmune screen came positive for high antinuclear antibodies (ANA) (titer $>1280$, confirmed twice) with a homogenous pattern, and the paraneoplastic/autoimmune panel was negative for anti amphiphysin, CV2, Ma2, NMDA, VGKC, Ri, Yo, and $\mathrm{Hu}$ antibodies.

In light of her high ANA and lack of improvement after 7 days on empirical treatment, a decision was made to start the patient on intravenous methylprednisolone at a dose of $1 \mathrm{~g}$ daily for 5 days. Early signs of improvement were evident on day five on IV steroids, where the patient started reproducing words and became oriented to time of the day and to her family, with less prominent agitation and irritability. The patient continued to improve over the following days and was discharged after 2 weeks. On her subsequent follow up a month later, she reported complete resolution of her symptoms and was fully independent and ready to resume her work, but she was unable to remember details from the early days of her admission. Follow-up EEG was done 3 months after discharge, this time using the US-made Natus Cadwell Easy 3 EEG (2016), which showed left-sided, mainly temporal, theta slowing, and less well-developed PDR in comparison with the right side (Fig. 4). Meanwhile, resolution of meningeal enhancement was noted on follow-up brain MRI with contrast done at the same period.

Nearly 2 years after her episode, biannual CT-NCAP is negative for malignancy, and the patient continues to follow up with her rheumatologist who opted for a watchful waiting strategy.

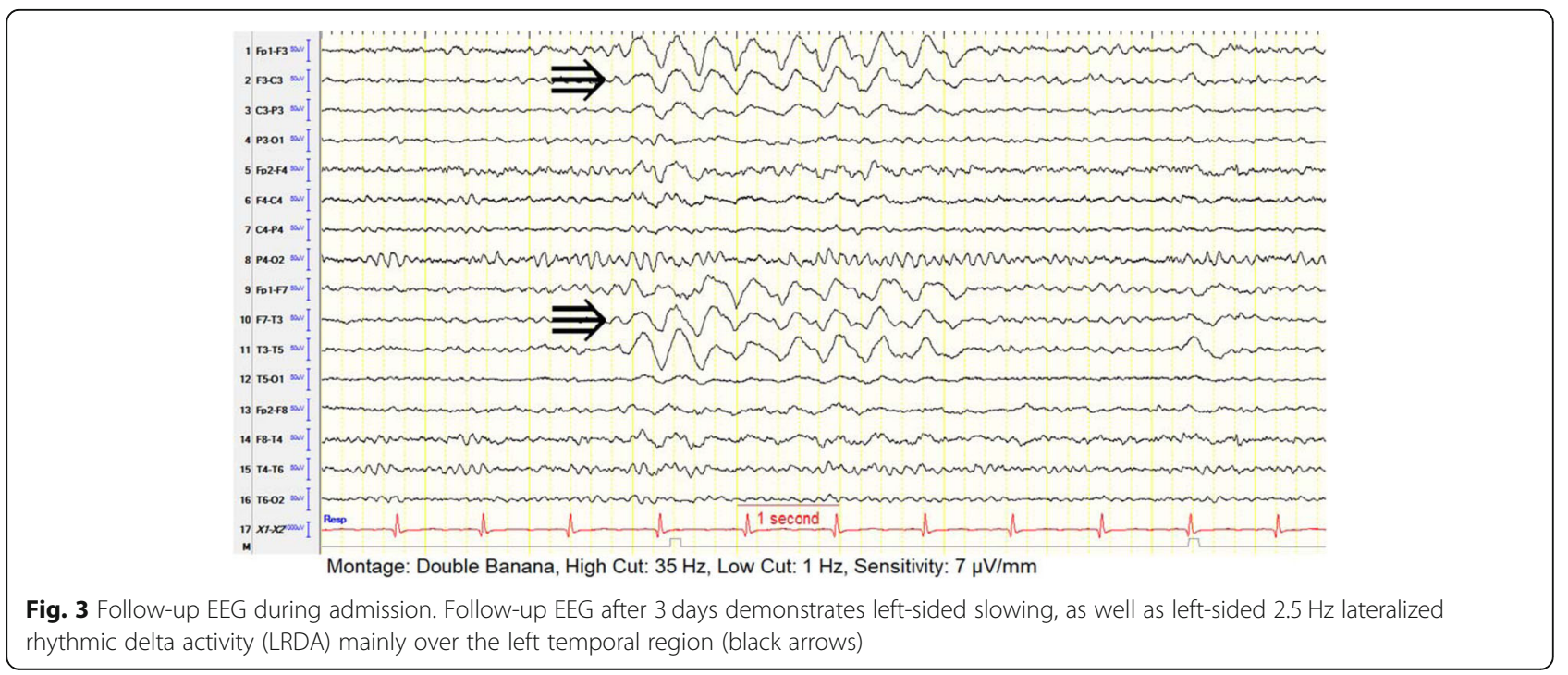




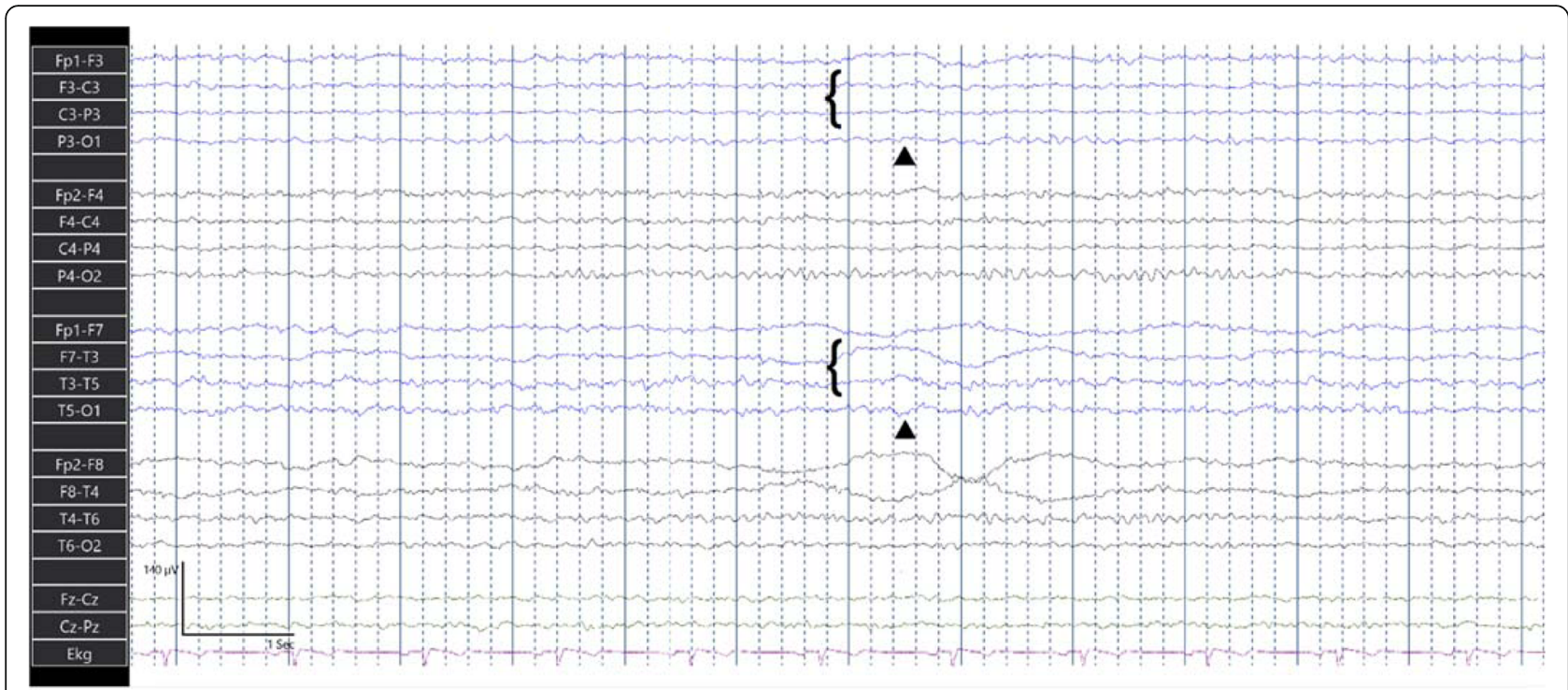

Montage: Double Banana, High Cut: $35 \mathrm{~Hz}$, Low Cut: $1 \mathrm{~Hz}$, Sensitivity: $7 \mu \mathrm{V} / \mathrm{mm}$

Fig. 4 Follow up EEG three months after discharge. Follow-up EEG 3 months after discharge demonstrates left-sided (brackets) theta slowing and less well-developed posterior dominant rhythm (PDR) (arrow heads). These changes marked a significant improvement in comparison with previous EEGs

\section{Discussion}

Encephalitis is a heterogeneous clinical entity with varying etiologies and clinical presentations. This and the lack of universal consensus over the diagnostic criteria result in over half of the cases remaining without a definitive etiology $[1,3,4]$. While infectious encephalitis is the most common [4], autoimmune encephalitis is increasingly recognized owing to a dramatic expansion over the last 15 years of a list of pathogenic neuronal antibodies. The discovery of these antibodies served the dual purpose of demonstrating the pathophysiology underlying neuronal damage and also acting as useful biomarkers defining different disease subtypes [8].

Until recently, diagnostic approaches for autoimmune encephalitis relied heavily on antibody testing and response to immunosuppressive treatment $[3,9,10]$. Technical and accessibility limitations associated with laboratory testing, and the inherently retrospective nature of these approaches, understandably impacted the chances of securing diagnoses in a timely manner and predisposed for treatment delays especially in subacute or vague cases, hence affecting patients' chances of a better outcome [2]. A widely received position paper by F Graus et al. in 2016 [2] attempted to tackle these limitations and facilitate prompt initiation of treatment by incorporating the clinical presentation early in the diagnostic algorithm. The paper set initial criteria for considering autoimmune encephalitis as possible; these criteria necessitated (1) identification of a compatible clinical syndrome, (2) reasonable exclusion of alternative etiologies, and (3) the presence of one or more of the following: focal central nervous system findings on examination, new-onset seizures, CSF pleocytosis, and abnormalities on MRI consistent with autoimmune encephalitis. Patients meeting these criteria are subsequently stratified into specific subcategories of probable or definite autoimmune encephalitis based on their antibody status and other criteria that include findings on neuroimaging, clinical assessment, CSF analysis, and occasionally EEG or brain biopsy findings.

Despite strong clinical suspicion, our patient did not fulfill the criteria for possible autoimmune encephalitis until after more than 2 weeks in the course of her illness, when subtle lateralized EEG findings prompted repetition of brain imaging that came positive. Ultimately, and although CSF-specific oligoclonal bands and CSF IgG index were not available and hence not tested, we believe that our case satisfies the criteria for the category designated by $\mathrm{F}$ Graus et al. as "autoantibody-negative but probable autoimmune encephalitis." This is supported by the very high ANA titer and the prompt response to pulse corticosteroids. An infectious etiology is much less likely giving the lack of clinical and laboratory markers of infection, the twice negative CSF workup, and the patient's lack of improvement after 7 days on empiric antibiotics and antivirals.

The fact that a definite etiology could not be pinpointed in our case correlates with the literature where demonstration of a definitive etiology is achieved in under half of encephalitis cases [1, 3, 4]. In addition, the components and methodology of autoimmune/paraneoplastic panels vary between countries and laboratories, 
and many antibodies lack sensitivity despite being fairly specific $[6,11]$. These limitations were among the reasons prompting the new criteria proposed by Graus et al. in the first place. Moreover, many entities within the family of autoimmune encephalitis remain rare and poorly understood. A relevant example is encephalitis due to anti-dipeptidyl-peptidase-like protein-6 (DPPX) antibodies, of which an estimated 40 cases only are reported in the literature [12, 13]. This encephalitis is often preceded by a diarrheal illness of varying duration and severity. Reports of response to immunosuppressing therapies are also variable, with some cases responding remarkably to a brief course of pulse steroids and others continuing to relapse or progress despite different and prolonged treatments [12]. Unfortunately, testing for DPPX antibodies is hardly available, and whether or not this particular encephalitis or another related or yet to be discovered encephalitis was causative in our case remains a mystery.

EEG findings in the setting of autoimmune encephalitis range from generalized slowing and dysfunction in a majority of cases to distinctive findings in some etiologies. Therefore, it is generally regarded as a highly sensitive but rarely specific investigation [2-4], which is reflected in the different diagnostic criteria where EEG is largely utilized for the diagnosis of seizures and nonconvulsive status epilepticus, or simply as a marker of a likely organic cause $[2,4,5]$. Still, the criteria acknowledge that EEG may occasionally detect patterns that are unique for certain forms of encephalitis [2]. An electrographic pattern named "extreme delta brush" is found in nearly $30 \%$ of NMDA encephalitis cases, and while actual specificity is still not precisely determined, this pattern is believed to have a prognostic value [14]. Moreover, in cases with mainly psychiatric symptoms, the value of EEG may lie in the absence of detectable changes, hence disfavoring organic causes [6]. Of note, throughout our supervision and later the revision phase of the patient data, we did not see high-frequency betarange brushes superimposed on the rhythmic delta activity in any of the EEGs. Despite that there might be intermittent low amplitude sharp component before or superimposed on delta waves, this does not fulfill the definition of delta brushes. Lateralized rhythmic delta activity with super-imposed sharp waves (LRDA+S) might be accepted instead of LRDA only.

EEG findings are also important in infectious encephalitis due to HSV, where focal or lateralized findings in the temporal or orbital frontal regions (such as focal epileptiform discharges, focal slowing, focally attenuated background activity, or PLEDs) are highly suggestive of herpes encephalitis and could predict outcome [15]. In addition, EEG plays an important role in the diagnosis of two neurodegenerative conditions that may be confused with autoimmune encephalitis: prion disease (for example Creutzfeldt-Jakob disease "CJD") and subacute sclerosing panencephalitis "SSPE". Advanced CJD is characterized by continuous, generalized, bisynchronous periodic sharp waves occurring at a frequency of $1-2 \mathrm{~Hz}$ $[16,17]$. SSPE is characterized on EEG by largeamplitude, periodic complexes that last up to $2 \mathrm{~s}$ and recur several times per minute $[18,19]$.

While we agree that the introduction and early utilization of the screening criteria for possible autoimmune encephalitis expedites diagnosis and treatment, these criteria only incorporated clinically evident newonset seizures, which might discourage investigators from the early deployment of EEG as a highly sensitive tool. Our case is a demonstration that EEG can be invaluable in the diagnosis of suspected autoimmune encephalitis, where even elusive and subtle findings as simple as voltage asymmetry helped direct further investigations and ultimately guided this serious diagnosis.

\section{Abbreviations}

HSV: Herpes simplex virus; CSF: Cerebrospinal fluid

EEG: Electroencephalography; CT: Computed tomography; CBC: Complete blood count; WBC: White blood cells; M. tb: Mycobacterium tuberculosis; EBV: Epstein-Barr virus; CMV: Cytomegalovirus; MRI: Magnetic Resonance Image; CT-NCAP: CT of the neck, chest, abdomen, and pelvis; PDR: Posterior dominant rhythm; LRDA: Lateralized rhythmic delta activity; ANA: Antinuclear antibodies; DPPX: Anti-dipeptidyl-peptidase-like protein-6; PLEDs: Periodic lateralized epileptiform discharges; CJD: Creutzfeldt-Jakob disease; SSPE: Subacute sclerosing panencephalitis

\section{Acknowledgements}

Not applicable.

\section{Authors' contributions}

BA participated in patient management and follow up, interpretation of images, data collection, literature review, writing of the initial draft, and preparation of the final manuscript and figures. KE contributed to interpretation of images, interpretation of EEGs, literature review, writing of the initial draft, and preparation and revision of the final manuscript. SA participated in patient management and follow up, data collection, literature review, writing of the initial draft and preparation of figures. DO participated in patient management and follow up, data collection, literature review, and writing of the initial draft. MQ participated in patient management and follow up, data collection, literature review, and writing of the initial draft MA participated in patient management, interpretation of images, literature review, writing of the initial draft, and revision of the final manuscript. AA contributed to patient follow up, literature review, and writing of the initial draft. AY participated in patient management and follow up, interpretation of images, interpretation of EEGs, supervised data collection, contributed to literature review and writing of the initial draft, and revised the final manuscript and figure legends. The author(s) read and approved the final manuscript.

\section{Authors' information}

KE is Professor of Neurology and Vice-President of Jordan University of Science and Technology. He ranked among the top publishing and highly cited researchers in the country in 2019 and remains the top cited in the field of adult neurology in Jordan. AY is consultant neurologist at King Abdullah University Hospital and Associate Professor of neurology at the Jordan University of Science and Technology faculty of medicine. MA is consultant neurologist at King Abdullah University Hospital and Associate Professor of neurology at the Jordan University of Science and Technology faculty of medicine. BA, SA, and DO are 4th year residents at the Neuroscience Department at King Abdullah University Hospital. MQ is a 5th year resident at 
the Neuroscience Department at King Abdullah University Hospital. AA is a 3rd year resident at the Department of Public Health and Family Medicine at King Abdullah University Hospital.

\section{Funding}

None.

\section{Availability of data and materials}

Available from Ahmed Yassin upon reasonable request.

\section{Ethics approval and consent to participate}

IRB approval is not required by the institution for case reports where sufficient anonymization of patient information is guaranteed.

\section{Consent for publication}

Patient consent for publication was obtained as necessary.

\section{Competing interests}

The authors declare that they have no competing interests.

\section{Author details}

'Department of Neuroscience, King Abdullah University Hospital (KAUH) of Jordan University of Science and Technology (JUST), P.O. Box 630001, Irbid 22110, Jordan. ${ }^{2}$ Department of Public Health and Family Medicine, King Abdullah University Hospital (KAUH) of Jordan University of Science and Technology (JUST), Irbid, Jordan.

Received: 6 March 2020 Accepted: 11 May 2020

Published online: 24 May 2020

\section{References}

1. Kumar R. Understanding and managing acute encephalitis. F1000Res. 2020 Jan 29;9. pii: F1000 Faculty Rev-60.

2. Graus F, Titulaer MJ, Balu R, Benseler S, Bien CG, Cellucci T, et al. A clinical approach to diagnosis of autoimmune encephalitis. Lancet Neurol. 2016; 15(4):391-404.

3. Venkatesan A, Tunkel AR, Bloch KC, Lauring AS, Sejvar J, Bitnun A, et al. Case definitions, diagnostic algorithms, and priorities in encephalitis: consensus statement of the international encephalitis consortium. Clin Infect Dis. 2013; 57(8):1114-28.

4. Britton PN, Eastwood K, Paterson B, Durrheim DN, Dale RC, Cheng AC, et al. Consensus guidelines for the investigation and management of encephalitis in adults and children in Australia and New Zealand. Intern Med J. 2015; 45(5):563-76.

5. Kneen R, Michael BD, Menson E, Mehta B, Easton A, Hemingway C, et al. Management of suspected viral encephalitis in children - Association of British Neurologists and British Paediatric Allergy, Immunology and Infection Group national guidelines. J Inf Secur. 2012;64(5):449-77.

6. Ganesh A, Wesley SF. Practice current: when do you suspect autoimmune encephalitis and what is the role of antibody testing? Neurol Clin Pract. 2018;8(1):67-73.

7. Solomon T, Michael BD, Smith PE, Sanderson F, Davies NW, Hart IJ, et al. Management of suspected viral encephalitis in adults--Association of British Neurologists and British Infection Association National Guidelines. J Inf Secur. 2012;64(4):347-73.

8. Dubey D, Pittock SJ, Kelly CR, McKeon A, Lopez-Chiriboga AS, Lennon VA, et al. Autoimmune encephalitis epidemiology and a comparison to infectious encephalitis. Ann Neurol. 2018;83(1):166-77.

9. Zuliani L, Graus F, Giometto B, Bien C, Vincent A. Central nervous system neuronal surface antibody associated syndromes: review and guidelines for recognition. J Neurol Neurosurg Psychiatry. 2012;83(6):638-45.

10. Graus F, Delattre JY, Antoine JC, Dalmau J, Giometto B, Grisold W, et al. Recommended diagnostic criteria for paraneoplastic neurological syndromes. J Neurol Neurosurg Psychiatry. 2004;75(8):1135-40.

11. Albadareen R, Gronseth G, Goeden M, Sharrock M, Lechtenberg C, Wang Y. Paraneoplastic autoantibody panels: sensitivity and specificity, a retrospective cohort. Int J Neurosci. 2017;127(6):531-8.

12. Hara M, Arino H, Petit-Pedrol M, Sabater L, Titulaer MJ, Martinez-Hernandez E, et al. DPPX antibody-associated encephalitis: main syndrome and antibody effects. Neurology. 2017;88(14):1340-8.
13. Boronat A, Gelfand JM, Gresa-Arribas N, Jeong HY, Walsh M, Roberts K, et al. Encephalitis and antibodies to dipeptidyl-peptidase-like protein-6, a subunit of Kv4.2 potassium channels. Ann Neurol. 2013;73(1):120-8.

14. Schmitt SE, Pargeon K, Frechette ES, Hirsch LJ, Dalmau J, Friedman D. Extreme delta brush: a unique EEG pattern in adults with anti-NMDA receptor encephalitis. Neurology. 2012;79(11):1094-100.

15. Kim YS, Jung KH, Lee ST, Kang BS, Yeom JS, Moon J, et al. Prognostic value of initial standard EEG and MRI in patients with herpes simplex encephalitis. J Clin Neurol. 2016;12(2):224-9.

16. Wieser HG, Schindler K, Zumsteg D. EEG in Creutzfeldt-Jakob disease. Clin Neurophysiol. 2006;117(5):935-51.

17. Andraus ME, Andraus CF, Alves-Leon SV. Periodic EEG patterns: importance of their recognition and clinical significance. Arq Neuropsiquiatr. 2012;70(2): 145-51.

18. Markand ON, Panszi JG. The electroencephalogram in subacute sclerosing panencephalitis. Arch Neurol. 1975;32(11):719-26.

19. Jafri SK, Kumar R, Ibrahim SH. Subacute sclerosing panencephalitis - current perspectives. Pediatric Health Med Ther. 2018;9:67-71.

\section{Publisher's Note}

Springer Nature remains neutral with regard to jurisdictional claims in published maps and institutional affiliations.

\section{Submit your manuscript to a SpringerOpen ${ }^{\circ}$ journal and benefit from:}

- Convenient online submission

- Rigorous peer review

- Open access: articles freely available online

High visibility within the field

- Retaining the copyright to your article

Submit your next manuscript at $\boldsymbol{\nabla}$ springeropen.com 